\title{
Polydopamine Coated Single-Walled Carbon Nanotubes as a Versatile Platform with Radionuclide Labeling for Multimodal Tumor Imaging and Therapy
}

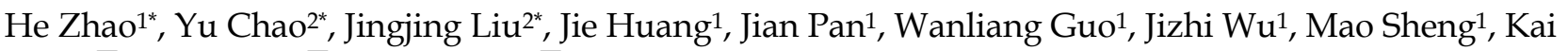 \\ Yang $^{3 凶}{ }^{\boxplus i a n ~ W a n g}{ }^{1 凶}$, Zhuang Liu ${ }^{2 \bowtie}$ \\ 1. Department of Pediatric Research, Children's Hospital affiliated to Soochow University, Suzhou, Jiangsu 215123, China \\ 2. Institute of Functional Nano \& Soft Materials (FUNSOM), Jiangsu Key Laboratory for Carbon-Based Functional Materials \& Devices, Soochow University, \\ Suzhou, Jiangsu 215123, China \\ 3. School of Radiation Medicine and Protection \& School for Radiological and Interdisciplinary Sciences (RAD-X), Collaborative Innovation Center of \\ Radiation Medicine of Jiangsu Higher Education Institutions, Medical College of Soochow University, Suzhou, Jiangsu 215123, China \\ * These authors contributed equally to this work. \\ $\bowtie$ Corresponding authors: wj196312@vip.163.com, kyang@suda.edu.cn, zliu@suda.edu.cn
}

(C) Ivyspring International Publisher. Reproduction is permitted for personal, noncommercial use, provided that the article is in whole, unmodified, and properly cited. See http://ivyspring.com/terms for terms and conditions.

Received: 2016.05.03; Accepted: 2016.06.19; Published: 2016.07.18

\begin{abstract}
Single-walled carbon nanotubes (SWNTs) with various unique properties have attracted great attention in cancer theranostics. Herein, SWNTs are coated with a shell of polydopamine (PDA), which is further modified by polyethylene glycol (PEG). The PDA shell in the obtained SWNT@PDA-PEG could chelate $\mathrm{Mn}^{2+}$, which together with metallic nanoparticulate impurities anchored on SWNTs offer enhanced both T1 and T2 contrasts under magnetic resonance (MR) imaging. Meanwhile, also utilizing the PDA shell, radionuclide ${ }^{131} \mid$ could be easily labeled onto SWNT@PDA-PEG, enabling nuclear imaging and radioisotope cancer therapy. As revealed by MR \& gamma imaging, efficient tumor accumulation of SWNT@PDA- ${ }^{131}$ I-PEG is observed after systemic administration into mice. By further utilizing the strong near-infarared (NIR) absorbance of SWNTs, NIR-triggered photothermal therapy in combination with ${ }^{131} \mathrm{I}$-based radioisotope therapy is realized in our animal experiments, in which a remarkable synergistic antitumor therapeutic effect is observed compared to monotherapies. Our work not only presents a new type of theranostic nanoplatform based on SWNTs, but also suggests the promise of PDA coating as a general approach to modify nano-agents and endow them with highly integrated functionalities.
\end{abstract}

Key words: Single-walled carbon nanotubes, Polydopamine, Multimodal imaging, Radiolabeling, Combined therapy

\section{Introduction}

In our fight against cancer, accurate diagnosis, effective therapy and precise assessment of prognosis, are all important to achieve the optimal treatment outcome. Nowadays, multimodal imaging that combines various imaging modalities to provide complementary information has demonstrated obvious advantages over single-modal imaging, promising for cancer diagnosis and prognostic evaluation.[1-4] On the other hand, combination therapy in which different treatment strategies are applied via a synergistic manner has become an important trend in the development of cancer treatment.[5-7] The design and fabrication of nanoscale platforms with integrated imaging and therapy functions would thus be demanded in the development of combination tumor therapy guided under multimodal imaging.

Carbon nanotubes (CNTs) including 
single-walled carbon nanotubes (SWNTs) with many unique physical and chemical properties have been extensively explored in the nanomedicine area.[8-11] It has been found that SWNTs exhibit intense Raman scattering, near-infrared (NIR) photoluminescence, strong photoacoustic signals and high T2 magnetic resonance (MR) contrast,[9] which together make SWNTs an excellent contrast agent based on their inherent properties for multimodal imaging. On the other hand, SWNTs with large surface area have been found to be efficient drug carriers for applications in drug delivery.[12, 13] Moreover, the strong NIR absorbance also makes SWNTs a robust photothermal agent for photothermal therapy (PTT) of cancer. $[9,10$, $14,15]$ In order to use CNTs for applications in biomedicine, their surface functionalization is critically important in regulating their behaviors in biological systems such as toxicity (non-functionalized raw CNTs could have toxic effects), pharmacokinetic profiles, and tumor targeting capability.[8, 9, 16] To date, a number of functionalization methods have been developed including covalent surface chemistry, non-covalent coating with amphiphilic molecules or polymers, or shelling with inorganic nanostructures such as mesoporous silica or gold shell.[9, 17, 18] However, among those methods, covalent surface modification would often damage the SWNT structure and affect their inherent optical properties; coating with amphiphilic molecules in many cases may have limited stability; while engineering with inorganic shells usually requires complicated procedures. $[9,10$, $17,19,20]$

Recently, polydopamine (PDA) derived from self-polymerization of dopamine has attracted intensive attention, not only as the nano-carrier by itself,[21-23] but also as a biocompatible coating to shell different types of functional nano-agents.[24, 25] In this work, a new CNT functionalization method is developed by coating SWNTs with a self-polymerized PDA shell, which not only makes SWNTs highly water soluble and stable, but also provides additional functionalities such as chelation with $\mathrm{Mn}^{2+}$ to offer enhanced T1 \& T2 MR contrast, as well as enabling efficient radioisotope labeling with ${ }^{131} \mathrm{I}$ to allow radioisotope therapy (RIT). With further modification with polyethylene glycol (PEG), SWNT@PDA-131-PEG with prolonged blood circulation half-life and efficient passive tumor targeting is obtained, as revealed by both in vivo MR imaging and ex vivo biodistribution based on gamma counting. By utilizing the inherent strong NIR absorbance of SWNTs, NIR-induced PTT is conducted with SWNT@PDA-131I-PEG to enhance its RIT, obtaining an obvious synergistic therapeutic outcome in a mouse tumor model. Our results present an interesting theranostic platform useful for multimodal imaging guided combination therapy of cancer, and suggest the great advantages of PDA coating as a general biocompatible surface functionalization strategy to engineer multifunctional nanostructures.

\section{Experimental Section}

Materials: dopamine, $\mathrm{MnCl}_{2}$, poly (maleic anhydride-alt-1-octadecene) and polyvinylpyrrolidone were purchased from Sigma-Aldrich and used as received. $\mathrm{NH}_{2}$-PEG (5 $\left.\mathrm{kDa}\right)$ polymer was obtained from PegBio, Suzhou, China. 4T1 murine breast cancer cell line was obtained from American Type Culture Collection (ATCC) and cultured at $37{ }^{\circ} \mathrm{C}, 5 \%$ $\mathrm{CO}_{2}$. RPMI-1640 culture medium was obtained from Hyclone. Female Balb/c mice were purchased from Nanjing Peng Sheng Biological Technology Co. Ltd.

Preparation of SWNT@PDA: SWNTs $(0.2$ $\mathrm{mg} / \mathrm{mL})$ were solubilized by PVP $(0.5 \mathrm{mg} / \mathrm{mL})$ using sonication for $5 \mathrm{~h}$,[26] yielding a black suspension which was then centrifuged at about $14000 \mathrm{rpm}$ for 1 $\mathrm{h}$ to remove the precipitates. The molar concentration of SWNTs (by assuming the length and diameter of SWNTs to be $150 \mathrm{~nm}$ and $1.5 \mathrm{~nm}$, respectively) was determined by the absorbance at $808 \mathrm{~nm}$ with a molar extinction co-efficient at $7.9 \times 10^{6} \mathrm{~cm}^{-1} \mathrm{M}^{-1}$.[27] To synthesis SWNT@PDA, the dopamine solution (0.4 $\mathrm{mg} / \mathrm{mL}$ ) was added into the SWNT/PVP dispersion $(0.1 \mathrm{mg} / \mathrm{mL})$ in Tris buffer $(10 \mathrm{mM}, \mathrm{pH} 8.5)$, sonicated for 5 minutes and stirred under air at room temperature for 20 h.[28] Then the SWNT@PDA sample was further purified by ultrafiltration (MWCO $=100 \mathrm{kDa}$ ) at $4000 \mathrm{rpm}$ for three times. The amount of PDA coating in the obtained SWNT@PDA was determined by comparing the total mass of freeze-dried SWNT@PDA sample versus the pre-determined net weight of SWNTs by its optical absorbance (at $808 \mathrm{~nm}$ ) and total volume of the solution before freeze-drying.

Synthesis of SWNT@PDA-PEG: PEG-grafted poly (maleic anhydride-alt-1-octadecene) (C18PMH-PEG) polymer was obtained according to previous reports.[10] SWNT@PDA $(0.6 \mathrm{mg} / \mathrm{mL})$ was mixed with C18PMH-PEG/ $\mathrm{H}_{2} \mathrm{O}(3 \mathrm{mg} / \mathrm{mL})$ and stirred for $4 \mathrm{~h}$ at room temperature. Then SWNT@PDA-PEG solution was centrifuged at 14800 rpm for 5 minutes to remove the precipitates.

Synthesis of $\mathbf{M n}^{2+}$ chelated SWNT@PDA-PEG: $0.24 \mathrm{~mL} \mathrm{MnCl} 2 / \mathrm{H}_{2} \mathrm{O}(10 \mathrm{mg} / \mathrm{ml})$ was added into the 1 mL SWNT@PDA-PEG $(0.6 \mathrm{mg} / \mathrm{mL})$ under stirring for $2 \mathrm{~h}$ at room temperature. The product SWNT@PDA-PEG/Mn was ultrafiltrated $($ MWCO = $100 \mathrm{kDa}$ ) at $4000 \mathrm{rpm}$ for three times. The $\mathrm{Mn}^{2+}$ concentration of SWNT@PDA-PEG/Mn was 
measured by inductively coupled plasma-mass spectrometry (ICP-MS) analysis.

Characterization: Transmission electron microscopy (TEM) imaging was carried out by using a FEI Tecnai F20 TEM at an acceleration voltage of 200 $\mathrm{kV}$. UV-Vis-NIR absorption spectra were measured by a GENESYS 10S UV-Vis spectrophotometer. The dynamic light scattering (DLS) and Zeta potential measurements were obtained from a Zetasizer Nano $Z$ (Malvern). Laser irradiation was used by an optical-fiber-coupled power-tunable diode laser (continuous wave) (maximal power $=10 \mathrm{~W}$, Hi-Tech Optoelectronics Co., Beijing, China).

${ }^{131}$ I radiolabeling of SWNT@PDA-PEG: ${ }^{131}$ I was labeled to the surface of SWNT@PDA-PEG by using a standard chloramine-T oxidation method according to the previous protocol.[22] Briefly, $0.5 \mathrm{~mL}$ SWNT@PDA-PEG $(0.6 \mathrm{mg} / \mathrm{mL}), 1 \mathrm{mCi}{ }^{131} \mathrm{I}$ and $0.2 \mathrm{~mL}$ chloramine- $\mathrm{T}(4 \mathrm{mg} / \mathrm{mL})$ were mixed together in $\mathrm{pH}$ 7.5 phosphate buffered saline (PBS) solution and reacted for 10 minutes at room temperature. The reacted solution was ultrafiltrated $(\mathrm{MWCO}=100 \mathrm{kDa})$ at $4000 \mathrm{rpm}$ to remove excess ${ }^{131} \mathrm{I}$. The ${ }^{131} \mathrm{I}$ radiolabeling efficiency on SWNT@PDA-PEG was determined by measuring the radioactivity of our yielded SWNT@PDA-PEG-131I by a gamma counter.

Cytotoxicity assays: $293 \mathrm{~T}$ cells were grown in DMEM culture medium with $10 \%$ fetal bovine serum (FBS) and $1 \%$ penicillin/streptomycin. 4T1 murine breast cancer cells were grown in RPMI-1640 culture medium with $10 \%$ FBS and $1 \%$ penicillin/streptomycin. To evaluate the potential toxicity at tested condition, 4T1 cells and 293T cells were seeded in 96-well plates at $1 \times 10^{4}$ /well and incubated with free ${ }^{131} \mathrm{I}$, SWNT@PDA-PEG and SWNT@PDA-131I-PEG at various concentrations for 24 h. Afterwards, the cells were washed with PBS. Then a standard methyl thiazolyltetrazolium (MTT, Sigma Aldrich) assay was performed to determine the relative cell viability.

In vitro combination therapy: $4 \mathrm{~T} 1$ cells were firstly incubated with SWNT@PDA-131I-PEG (80 nM of SWNTs, corresponding to $150 \mu \mathrm{Ci}$ of ${ }^{131} \mathrm{I}$ ) for $24 \mathrm{~h}$, and then irradiated by an $808 \mathrm{~nm}$ NIR laser at the

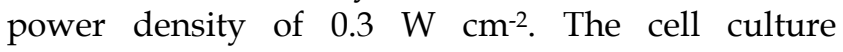
temperature was kept at about $46^{\circ} \mathrm{C}$ for $20 \mathrm{~min}$. Then the relative cell viabilities were measured by the MTT assay. Meanwhile, the cells at the same therapy conditions were stained with Calcine AM and prodium iodide (PI) for $30 \mathrm{~min}$ to differentiate live (green color) and dead cells (red color). After PBS washing, the cells were observed by a fluorescence microscope (Olympus, IX73).

In vivo combination therapy: All animal experiments were performed under protocols approved by Soochow University Laboratory Animal Center. The tumor model was created by subcutaneous injection of $1 \times 10^{6} 4 \mathrm{~T} 1$ cells into the back of each mouse. When the tumor volumes reached about $50 \mathrm{~mm}^{3}$, mice were randomly divided into six groups with five mice per group including untreated group, mice i.v. injected with free ${ }^{131} \mathrm{I}$ (200 $\mu \mathrm{Ci})$, SWNT@PDA-PEG with and without laser irradiation ([SWNT]:4.3 mg kg-1), and SWNT@PDA131I-PEG with and without laser irradiation ([SWNT]:4.3 $\mathrm{mg} \mathrm{kg}^{-1}, 200 \mu \mathrm{Ci}$ ). After $24 \mathrm{~h}$, the tumors treated with SWNT@PDA-PEG and SWNT@PDA-131IPEG were irradiated by the $808 \mathrm{~nm}$ NIR laser at a power density of $0.6 \mathrm{~W} \mathrm{~cm}^{-2}$ to maintain the tumor temperature at about $46{ }^{\circ} \mathrm{C}$ for $20 \mathrm{~min}$ under the monitoring by the infrared camera (Infrared Cameras. Inc.). After treatments, the tumor sizes and body weights were measured every 2 days for 2 weeks. The tumor volume was calculated according to the following formula: volume $=$ width $^{2} \times$ length $/ 2$. Relative tumor volumes were calculated as $\mathrm{V} / \mathrm{V}_{0}\left(\mathrm{~V}_{0}\right.$ was the initial tumor volume when the treatment began). Then the tumors were collected and weighed after 14 day post injection. On the other hand, tumors slices from all groups were collected 1 day post treatment for hematoxylin and eosin (H\&E) and terminal deoxynucleotidyl transferase-mediated dUTP-biotin nick end labeling assay (TUNEL) staining (Roche) and observed by a digital microscope (Leica QWin). Next, the major organs including heart, liver, kidneys, lung and spleen were harvested from untreated group and SWNT@PDA-131I-PEG with laser irradiation group, fixed in $4 \%$ of formalin, embedded in paraffin, and sectioned into $4 \mu \mathrm{m}$ slices for $\mathrm{H} \& \mathrm{E}$ staining.

Multimodal imaging: Mice bearing 4T1 tumor were i.v. injected with SWNT@PDA-131I-PEG ([SWNT]: $4.3 \mathrm{mg} / \mathrm{kg}, 200 \mu \mathrm{Ci}$ per mouse) for in vivo IR thermal, gamma and MR imaging. IR thermal imaging was irradiated with $808 \mathrm{~nm}$ laser at a power density of $0.6 \mathrm{~W} / \mathrm{cm}^{2}$ for $20 \mathrm{~min}$ and imaged using an IR thermal camera (Infrared Cameras. Inc.). Gamma imaging was accomplished with in vivo animal imaging system (Kodak, FX Pro). 4T1 tumor-bearing mice were i.v. injected with SWNT@PDA-PEG/Mn ([SWNT]: $4.3 \mathrm{mg} / \mathrm{kg}$, [Mn]: $1.61 \mathrm{mg} / \mathrm{kg}$ ) for in vivo MR imaging, which was performed with the $3 \mathrm{~T}$ clinical MRI scanner (Bruker Biospin Corporation, Billerica, MA, USA) equipped with a special coil for small animal imaging. The MR images of SWNT@PDA-PEG and SWNT@PDA-PEG/Mn were collected at the same time, using a $3 \mathrm{~T}$ clinical MR scanner. After acquiring MR images, the signal intensity values were measured for each sample in the interest region. 
Blood circulation and biodistribution of SWNT@PDA-131I-PEG: Balb/c mice were i.v. injected with SWNT@PDA-131I-PEG ([SWNT]:4.3 mg/kg, 200 $\mu \mathrm{Ci}$ of $\left.{ }^{131} \mathrm{I}\right) .10 \mu \mathrm{L}$ blood was obtained at different time, and measured by the gamma counter (Science and Technology Institute of China in Jia Branch Innovation Co., Ltd.). Major organs including liver, spleen, kidney, heart, lung, skin, muscle, bone and tumor were collected at $24 \mathrm{~h}$ post injection and measured by the gamma counter.

\section{Results and Discussion}

The strategy for the synthesis of PDA coated SWNTs and the subsequent PEG modification is shown in Figure 1a. Raw SWNTs produced by the high-pressure $\mathrm{CO}$ disproportionation method (Hipco)
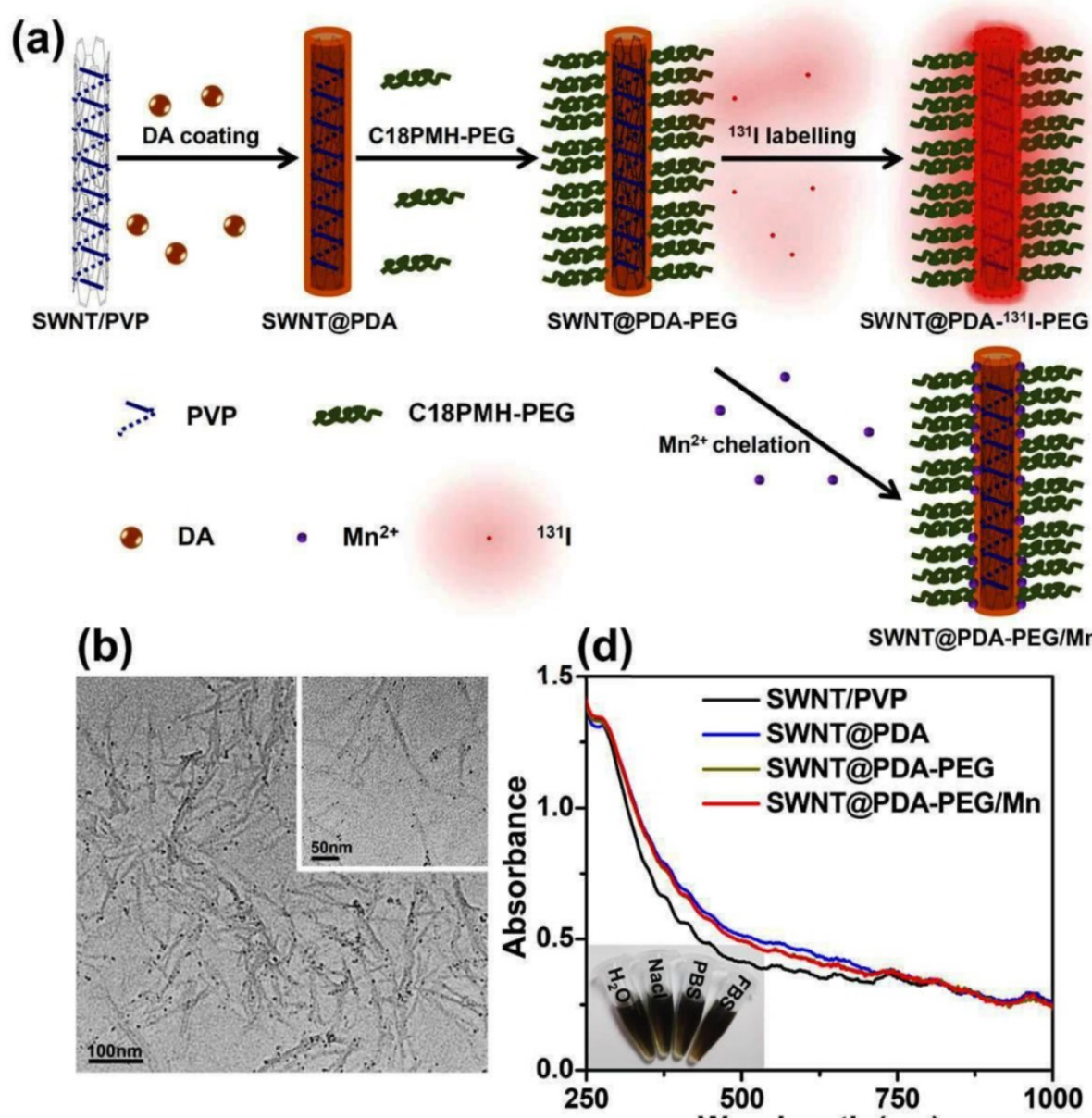

(c)
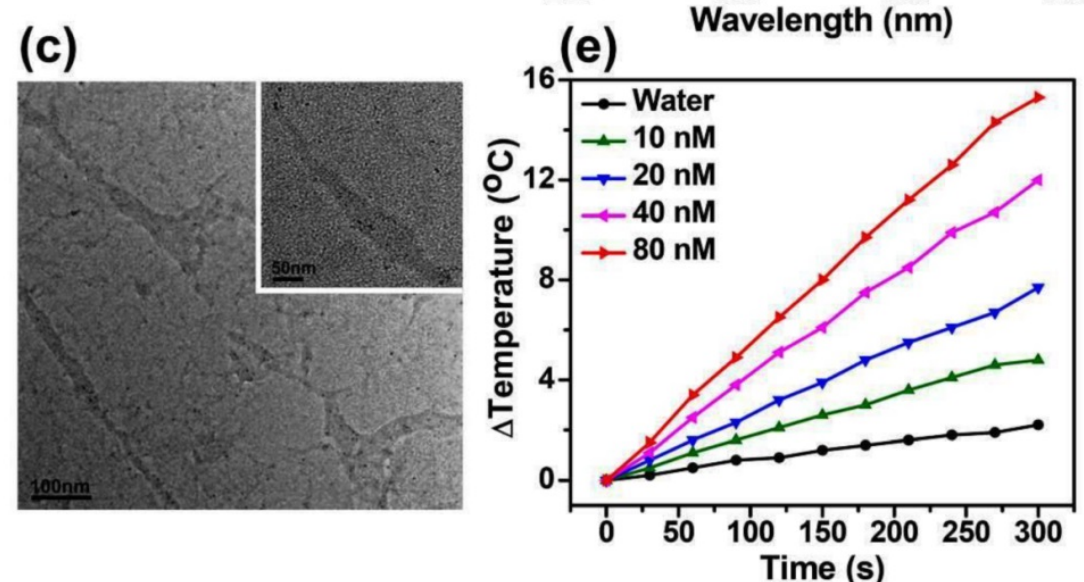

Figure 1. Preparation and characterization of SWNT@PDA-PEG. (a) A schematic illustration for the fabrication of SWNT@PDA-PEG. (b\&c) TEM images of SWNT/PVP (b) and SWNT@PDA (c). Inset is a TEM image with higher resolution. (d) UV-Vis-NIR absorbance spectra of SWNT/PVP, SWNT@PDA, SWNT@PDA-PEG and SWNT@PDA-PEG/Mn solutions at the same SWNT concentration. Inset: a photo of SWNT@PDA-PEG/Mn in various types of physiological buffers. (e) Temperature change curves of SWNT@PDA-PEG solution at different concentrations (i.e. 10, 20, 40, $80 \mathrm{nM}$ ) exposed to the $808 \mathrm{~nm}$ laser with a power density of $0.7 \mathrm{~W}$ cm 2 for 5 min. The molar concentrations of SWNTs were determined by a literature method.[27] 
were firstly solubilized by polyvinylpyrrolidone (PVP). When dopamine was added into the SWNT/PVP solution under an alkaline $\mathrm{pH}$, it was self-polymerized to form PDA coated on the surface of SWNTs. Then, the yielded SWNT@PDA was modified with PEG-grafted poly (maleic anhydride-alt-1-octadecene)

(C18PMH-PEG) synthesized following the previously reported protocol to obtain PEGylated SWNT@PDA (SWNT@PDA-PEG).[10] Transmission electron microscopy (TEM) images (Figure 1b, 1c and Supporting Information Figure S1) of SWNT samples revealed that a PDA shell was formed around SWNTs and $\mathrm{Mn}^{2+}$ had no effect on the structure of SWNT@PDA-PEG. With PEG modification, SWNT@PDA-PEG showed great dispersity in various types of physiological buffers (Figure 1d, inset). Owing to the strong NIR absorbance of SWNTs (Figure 1d), SWNT@PDA-PEG was found to be a more efficient photothermal heating agent than bare PDA once exposed to an 808-nm NIR laser (Figure 1e, Supporting Information Figure S2).

As a powerful imaging modality, magnetic resonance (MR) imaging is extensively used in the clinic. In many previous studies, it has been demonstrated that metallic nanoparticles anchored on SWNTs formed from the added catalysts during their synthesis could offer contrast in $\mathrm{T}_{2}$-weighted $\mathrm{MR}$ imaging.[9] It is also known that PDA with hydroxyl groups would form chelates with different types of metal ions.[21, 29] Since paramagnetic $\mathrm{Mn}^{2+}$ with five unpaired $3 \mathrm{~d}$ electrons is known to be a great contrast agent for $\mathrm{T}_{1}$-weighted $\mathrm{MR}$ imaging and may also enhance the $\mathrm{T}_{2}-\mathrm{MR}$ contrast, $[30-34]$ we thus wondered if SWNT@PDA-PEG could be further loaded with $\mathrm{Mn}^{2+}$ to simultaneously allow $\mathrm{T}_{1} / \mathrm{T}_{2} \mathrm{MR}$ imaging. By simply mixing $\mathrm{MnCl}_{2}$ with SWNT@PDA-PEG, $\mathrm{Mn}^{2+}$-chelated SWNT@PDA-PEG (SWNT@PDAPEG/Mn) was obtained. The increased zeta potential indicated the successful loading of $\mathrm{Mn}^{2+}$ ions in the obtained SWNT@PDA-PEG/Mn (Supporting Information Figure S1), in which the mass ratio of SWNT : $\mathrm{Mn}^{2+}$ was measured to be $1: 0.374$ by Inductively Coupled Plasma-Mass Spectrometry (ICP-MS). Interestingly, compared to SWNT@PDA-PEG without chelation of $\mathrm{Mn}^{2+}$, our SWNT@PDA-PEG/Mn samples showed greatly enhanced concentration-dependent brightening and darkening effects as observed by both $T_{1^{-}}$and $\mathrm{T}_{2}$-weighted MR imaging, respectively (Figure $2 \mathrm{a}, 2 \mathrm{~b}$ ), demonstrating that the chelation of $\mathrm{Mn}^{2+}$ could remarkably enhance both $\mathrm{T}_{1^{-}}$and $\mathrm{T}_{2}$-contrasts of SWNTs under MR imaging (Figure 2a, 2b). The corresponding enhanced relaxivities $\left(r_{1}\right.$ and $\left.r_{2}\right)$ of SWNT@PDA-PEG/Mn by chelation of $\mathrm{Mn}^{2+}$ (compared to SWNT@PDA-PEG without $\mathrm{Mn}^{2+}$ ) were determined to be $r_{1}=3.98 \mathrm{mM}^{-1} \mathrm{~s}^{-1}$ and $\mathrm{r}_{2}=84.61$ $\mathrm{mM}^{-1} \mathrm{~s}^{-1}$, as calculated by plotting $1 / \Delta \mathrm{T} 1$ and $1 / \Delta \mathrm{T} 2$ against $\mathrm{Mn}^{2+}$ concentrations, respectively $(\Delta \mathrm{T} 1 \& \Delta \mathrm{T} 2$ are the relaxation time differences between SWNT@PDA-PEG/Mn and SWNT@PDA-PEG at the same SWNT concentration). This integrated $\mathrm{T}_{1^{-}}$and $\mathrm{T}_{2}$-weighted MR modalities could potentially offer more complete and accurate information about the lesion by overcoming the shortcomings of each single MR mode.[30, 35] (a)

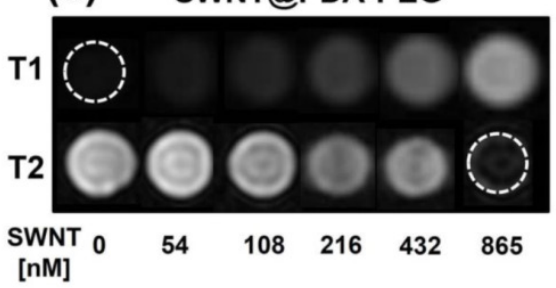

(c)

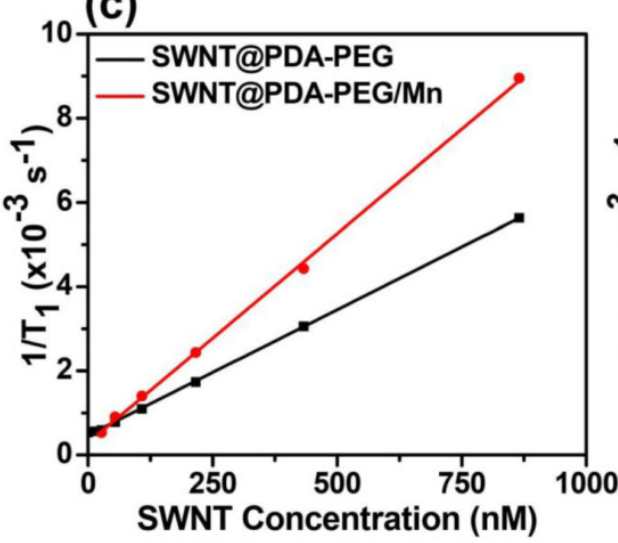

(b) SWNT@PDA-PEG/Mn
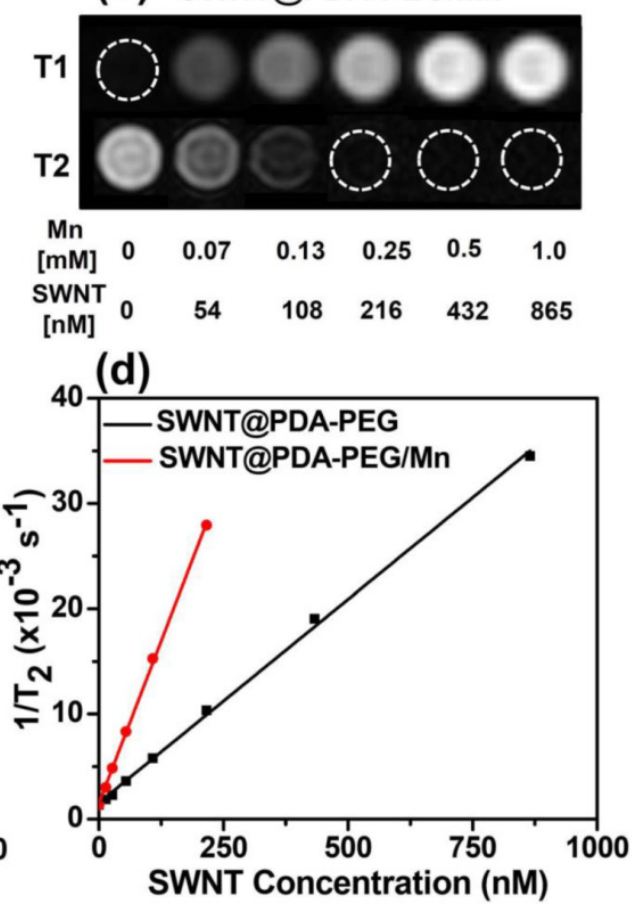

Figure 2. MR imaging of SWNT@PDA-PEG samples without or with chelation of $\mathrm{Mn}^{2+}$. (a\&b) $\mathrm{T}_{1}$ - and $\mathrm{T}_{2}$ - weighted MR images of SWNT@PDA-PEG samples before (a) and after (b) $\mathrm{Mn}^{2+}$ chelating. The SWNT concentrations varied from 0 to 865 $\mathrm{nM}$, corresponding to $\mathrm{Mn}$ concentrations from 0 to $1 \mathrm{mM}$ (for SWNT@PDA-PEG/Mn). (c\&d) Tl (c) and T2 (d) relaxation rates of SWNT@PDA-PEG and SWNT@PDA-PEG/Mn at different SWNT concentrations. 
(a)

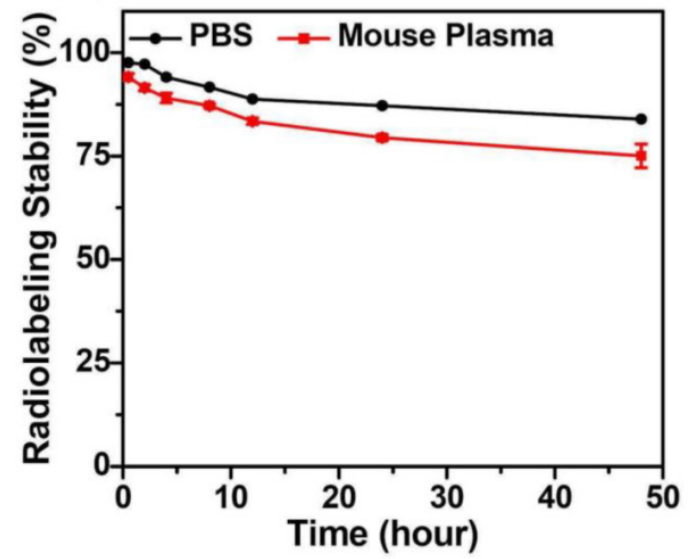

(c)

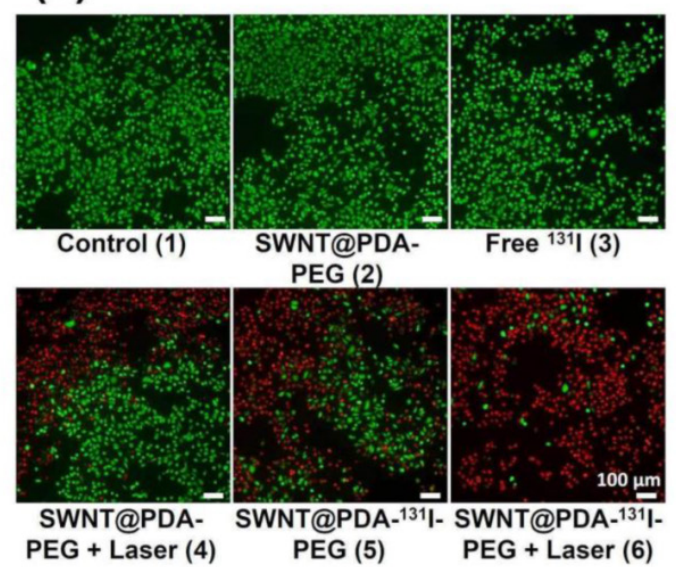

(b)

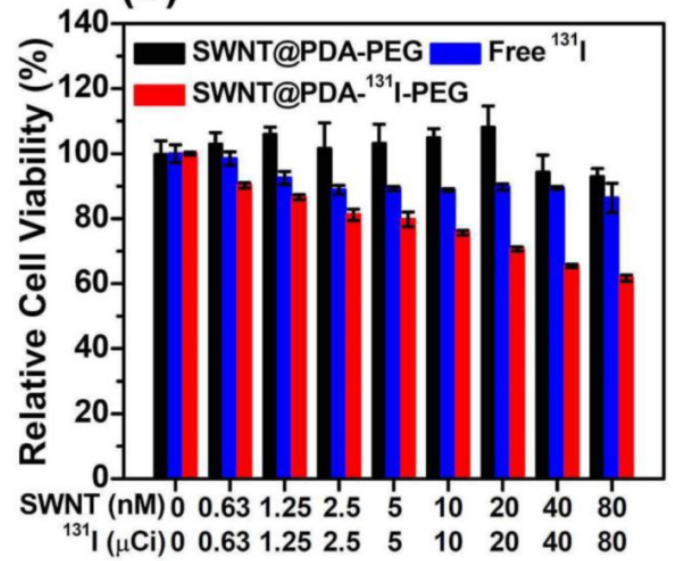

(d)

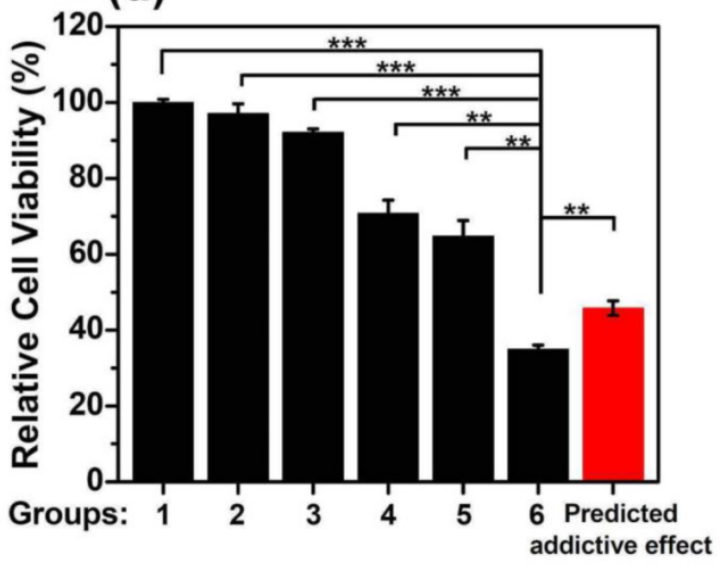

Figure 3. In vitro cell experiments. (a) The radiolabeling stability of SWNT@ @PDA-13I|-PEG incubated with PBS and mouse plasma at $37^{\circ} \mathrm{C}$. (b) The relative viabilities of $4 T 1$ cells incubated with various concentrations of SWNT@PDA-PEG, free 131/ and SWNT@PDA-|31|-PEG. For SWNT@PDA-PEG and SWNT@PDA-131|-PEG, SWNT concentrations varied from 0 to $80 \mathrm{nM}$. For free 131I and SWNT@PDA-131I-PEG, 131/ concentrations varied from 0 to $80 \mu \mathrm{Ci}$. (c\&d) The fluorescence imaging of Calcein AM/PI stained 4T1 cells (c) and their relative viabilities (d) after different treatments. Group 1: Control; Group 2: SWNT@PDA-PEG; Group 3: Free 131!; Group 4: SWNT@PDA-PEG + Laser; Group 5: SWNT@PDA-13II-PEG; Group 6: SWNT@PDA-13II-PEG + Laser. The predicted addictive effect was calculated by multiplying the remained cell viability ratio in group 4 (PTT only) with that in group 5 (RIT only). The concentration of $131 \mid$ was $150 \mu \mathrm{Ci}$ (corresponding to 80 nM of SWNT@PDA-PEG in the SWNT@PDA-131|-PEG sample). The laser irradiation was conducted at about $0.3 \mathrm{~W} \mathrm{~cm}{ }^{-2}$ to maintain the temperature at about $46 \circ \mathrm{C}$ for 20 min. $\mathrm{P}$ values were calculated by the Student's two-tailed t-test (*** $\mathrm{p}<0.001$ or $* * \mathrm{p}<0.01)$.

In our previous work, it was found that PDA with phenol groups could be easily labeled with radioactive iodine (e.g. ${ }^{131}$ I) with rather high efficiency via a standard chloramine-T oxidation method.[22] Using a similar method, SWNT@PDA-PEG was labeled by ${ }^{131} \mathrm{I}$ by mixing with $\mathrm{K}^{131} \mathrm{I}$ and chloramine- $\mathrm{T}$ for 10 minutes, achieving a labeling yield as high as $\sim 90 \%$.[22] Notably, the ratio of SWNT : ${ }^{131}$ I in the final SWNT@PDA-131I-PEG could be easily tuned based on the requirement of different experiments, by simply changing on the feeding ratio during radiolabeling. We then studied the radiolabeling stability of SWNT@PDA-131I-PEG by incubating those radioactive nanotubes in phosphate buffer saline (PBS) and mouse plasma at $37{ }^{\circ} \mathrm{C}$. It was found that SWNT@PDA-131I-PEG exhibited good stability in mouse plasma and PBS over $48 \mathrm{~h}$ (Figure 3a).

Next, the potential toxicity of the nanoplatform was tested by a standard methyl thiazolyltetrazolium
(MTT) assay. As expected, the cytotoxicity of SWNT@PDA-PEG without radiolabeling was not observed when the cells were incubated with different concentrations of SWNT@PDA-PEG for 24 h (Figure $3 \mathrm{~b}$ and Supporting Information Figure S3). On the other hand, SWNT@PDA-131I-PEG was able to effectively inhibit cell growth by a concentration-dependent manner (Figure $3 b$ and Supporting Information Figure S3). Compared to free 131I, SWNT@PDA-131I-PEG with the same radioactivity dose showed enhanced killing efficacy (Figure $3 \mathrm{~b}$ and Supporting Information Figure S3).

The combination of RIT and PTT was studied in vitro with such multifunctional SWNT@PDA-131I-PEG nanoplatform. In our experiments, 4T1 cells were incubated with SWNT@PDA-131I-PEG (80 nM of SWNT@PDA-PEG, $150 \mu \mathrm{Ci}$ of $\left.{ }^{131} \mathrm{I}\right)$ for $24 \mathrm{~h}$, irradiated by an $808-\mathrm{nm}$ laser $\left(0.3 \mathrm{~W} \mathrm{~cm}^{-2}, 20 \mathrm{~min}\right)$. In contrast to RIT alone (SWNT@PDA-131I-PEG without laser) and 
PTT alone (SWNT@PDA-PEG with laser), which could partially induce cancer cell death at the tested dose, the combined RIT and PTT (SWNT@PDA-131I-PEG with laser) offered greater killing efficacy, as revealed by both the relative cell viability data and fluorescence images of Calcine AM and propidium iodide (PI) co-stained cells (Figure 3c, $3 \mathrm{~d})$. At the same time, the actual synergistic efficacy induced by RIT \& PTT combination (Group 6) was better than the predicted addictive effect (Figure 3d). Therefore, the combined RIT and PTT with SWNT@PDA-131I-PEG could offer a remarkable synergistic killing activity in vitro on cancer cells.

The multimodal imaging and in vivo behaviors of SWNT@PDA-PEG were then studied. For in vivo MR imaging, we evaluated the contrast enhancing effect in Balb/c mice bearing 4T1 tumors with i.v. injection of SWNT@PDA-PEG/Mn. After 24 hours i.v. injection with SWNT@PDA-PEG/Mn, the tumor contrast became brighter under $\mathrm{T}_{1}$-weighted $\mathrm{MR}$ imaging, showing marked positive enhancement, while in $\mathrm{T}_{2}$-weighted MR image, the tumor became darker and showed an obvious negative contrast (Figure 4a).

(b)

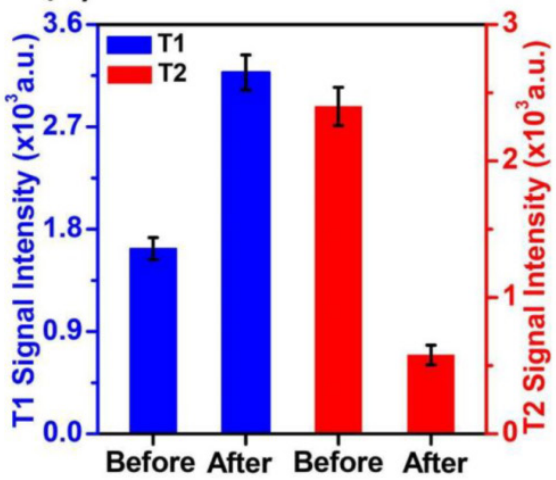

(c)
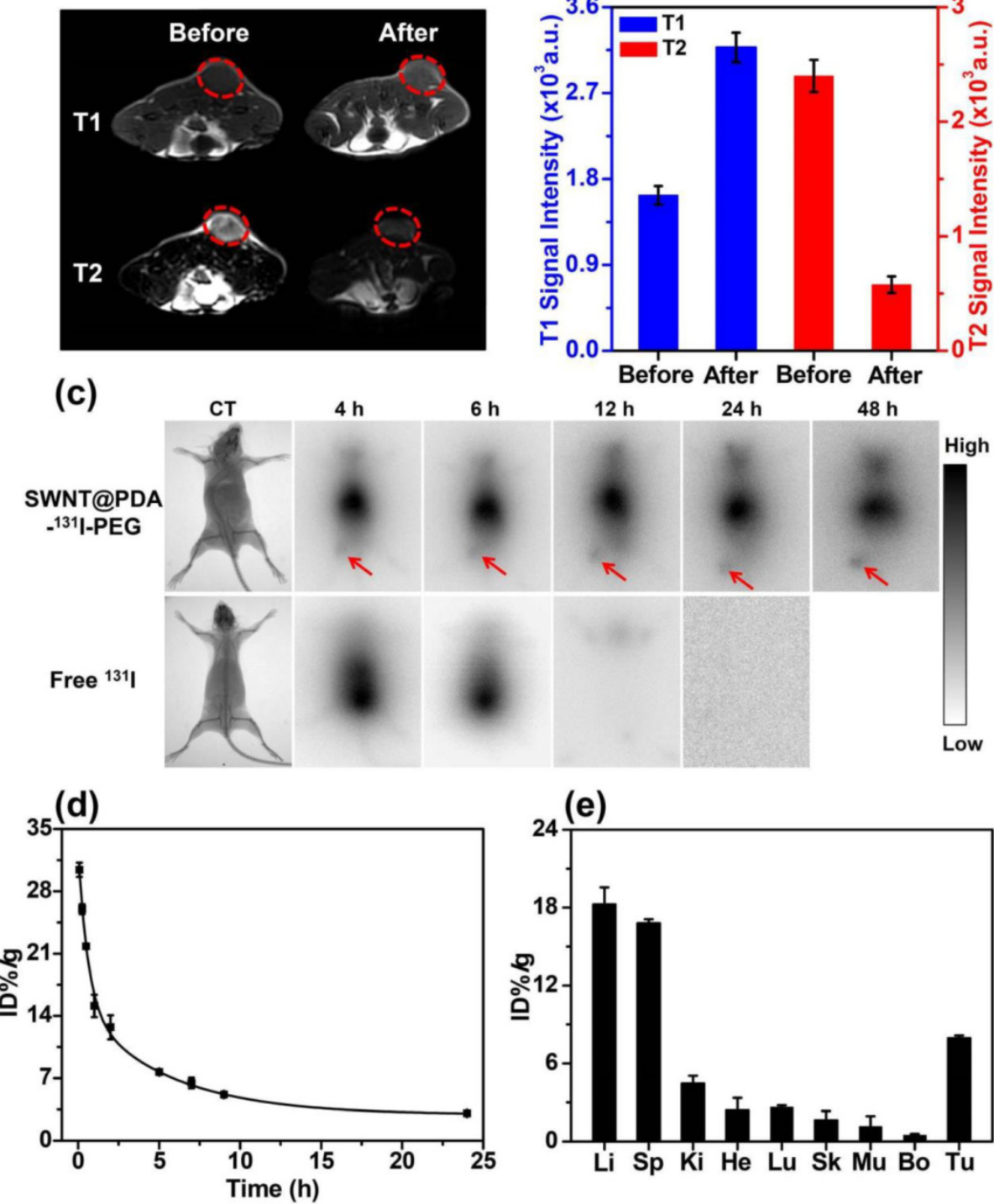

(e)

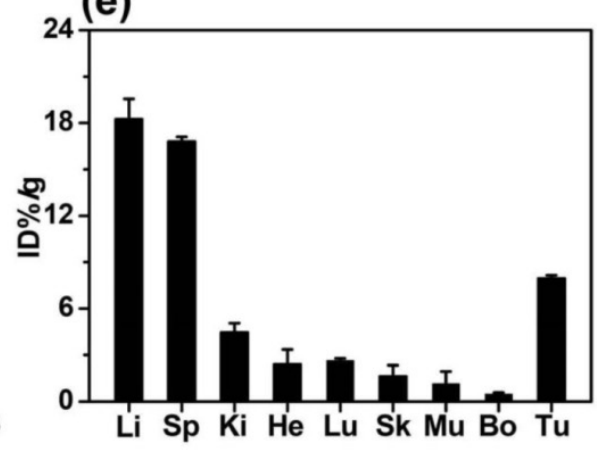

Figure 4. In vivo behaviors of SWNT@PDA-13II-PEG. (a) In vivo $T_{1}$ - (upper) and $T_{2}$-weighted MR images (bottom) of a 4T1 tumor-bearing mouse taken before injection (left) and 24 h post injection (right) of SWNT@PDA-PEG/Mn. Obvious brightening and darkening effects showed up in the tumor after i.v. injection of SWNT@PDA-PEG/Mn by T1and $T_{2}$-weighted MR imaging, respectively. (b) $T_{1}$ - (left) and $T_{2}$-weighted (right) MR signals intensities of the tumor before and 24 h post i.v. injection of SWNT@PDA-PEG/Mn, which offered strong tumor contrasts under both $T_{1}$ - and $T_{2}$-weighted MR imaging modes. (c) Gamma imaging of SWNT@PDA-13II-PEG-treated mice and free 131I-treated mice. Notably, free 131! was completely excreted after 12 h, while SWNT@PDA-131|-PEG showed obvious tumor accumulation. (d) The blood circulation of SWNT@PDA-131/-PEG after i.v. injection determined by gamma-counting. (e) The biodistribution of SWNT@PDA-131I-PEG in 4T1 tumor-bearing mice measured at 24 h p.i. Li: liver; Sp: spleen; Ki: kidney; He: heart; Lu: lung; Sk: skin; Mu: muscle; Bo: bone; Tu: tumor. 
Using region-of-interest (ROI) quantification method, the quantitative MR imaging results further demonstrated the $T_{1}$ signal intensity was obviously increased while the $T_{2}$ signal intensity was decreased in the tumor upon i.v. injection with SWNT@PDA-PEG/Mn (Figure 4b), suggesting the efficient tumor accumulation of those nanotubes. ${ }^{131} \mathrm{I}$ with gamma emission could offer contrast under single photon emission computed tomography (SEPCT).[22] Due to our limited access to small animal SPECT, gamma imaging was conducted as a proof-of-concept experiment to image 4T1 tumor-bearing mice after i.v. injection with either free 131I or SWNT@PDA-131I-PEG ([SWNT]: $4.3 \mathrm{mg} / \mathrm{kg}$, 200 $\mu \mathrm{Ci}$ of $\left.{ }^{131} \mathrm{I}\right)$. After $6 \mathrm{~h}$, most of free ${ }^{131} \mathrm{I}$ could be rapidly excreted from the mouse body. In contrast, SWNT@PDA-131I-PEG could accumulate in the tumor after $4 \mathrm{~h}$ and become clear after $12 \mathrm{~h}$ (Figure $4 \mathrm{c}$ and Supporting Information Figure S4). A contrast agent with integrated MR and gamma imaging could present more comprehensive information at a single-dose.

Next, blood circulation and biodistribution of SWNT@PDA-131I-PEG were studied in vivo. After i.v. injection of SWNT@PDA-131I-PEG ([SWNT]:4.3 $\mathrm{mg} / \mathrm{kg}, 200 \mu \mathrm{Ci}$ of $\left.{ }^{131} \mathrm{I}\right)$, blood was sampled from Balb/c mice at various time points post-injection (p.i.). The concentrations of nanotubes in the blood were detected by the radioactivity. As shown in Figure 4d, while the blood levels of SWNT@PDA-131I-PEG decreased gradually over time, the ${ }^{131}$ I signals in the blood remained at a relatively high level even at $24 \mathrm{~h}$ p.i.. The blood circulation curve revealed that the pharmacokinetics of SWNT@PDA-131I-PEG followed a two-compartment model. The first and second phase blood half-lives of SWNT@PDA-131I-PEG were calculated to be $t_{1 / 2}(\alpha)=0.932 \pm 0.124 h$ and $t_{1 / 2}(\beta)=$ $15.046 \pm 0.412 \mathrm{~h}$. For the biodistribution measurement, the main organs and tumors in tumor-bearing mice were collected $24 \mathrm{~h}$ i.v. injection with SWNT@PDA-131I-PEG ([SWNT]:4.3 mg/kg, $200 \mu \mathrm{Ci}$ of $\left.{ }^{131} \mathrm{I}\right)$. The radioactivity levels in different tissues were measured by a gamma counter. It was found that the radioactivity signal in the tumor was higher than that in other organs except liver and spleen, which were reticuloendothelial systems (RES) responsible for the clearance of foreign nanoparticles from the blood (Figure 4e).

Furthermore, we studied the in vivo treatment effect of RIT \& PTT combination therapy with SWNT@PDA-131I-PEG under the inspiration of the observed synergistic therapeutic effect in vitro and the efficient in vivo tumor accumulation of SWNT@PDA-131I-PEG. The tumor model was developed by subcutaneously injecting $1 \times 10^{6}$ murine breast cancer $4 \mathrm{~T} 1$ cells into the back of each Balb/c mouse.[9] When the tumor volume reached about 50 $\mathrm{mm}^{3}$, mice were randomly divided into six groups $(6$ mice in each group): Group 1: PBS + NIR; Group 2: SWNT@PDA-PEG; Group 3: Free 131I; Group 4: SWNT@PDA-PEG + NIR; Group 5: SWNT@PDA131I-PEG; Group 6: SWNT@PDA-131I-PEG + NIR. The mice were i.v. injected with various agents ([SWNT]:4.3 mg/kg, $200 \mu \mathrm{Ci}$ of ${ }^{131} \mathrm{I}$ ) in certain groups. After $24 \mathrm{~h}$ p.i., the tumors in Group 1, 4 and 6 were irradiated by the $808 \mathrm{~nm}$ laser at a power density of $0.6 \mathrm{~W} \mathrm{~cm}^{-2}$. As monitored by the IR thermal camera (Figure 5a, 5b), the tumor temperatures of mice treated with SWNT@PDA-PEG and SWNT@PDA131I-PEG rapidly increased to about $46{ }^{\circ} \mathrm{C}$ and maintained at that level during laser irradiation. In comparison, the mice treated with PBS exhibited much less significant heating in the tumor under laser irradiation, and the final temperature was about 31 ${ }^{\circ} \mathrm{C}$.

After different treatments, we measured the tumor sizes every 2 days. Remarkably, the tumors in Group 6 with injection of SWNT@PDA-131I-PEG were completely eliminated after NIR laser irradiation as a result of the combined RIT and PTT (Figure 5c, 5d, 5e and Supporting Information Figure S4). In contrast, neither PTT alone (Group 4: SWNT@PDA-PEG + NIR) nor RIT alone (Group 5: SWNT@PDA-131I-PEG) could completely inhibit the tumor growth, although the tumor development was partly delayed. Notably, RIT alone in Group 5 with SWNT@PDA-131I-PEG showed better efficacy than free ${ }^{131}$ I in Group 3, likely owing to the enhanced tumor uptake for ${ }^{131}$ I loaded on nanotubes. At 14 days p.i., the tumors from all the groups were collected and weighed. The averaged tumor weights were well consistent with the measured tumor growth curves (Figure $5 \mathrm{~d}$ and $5 \mathrm{e}$ ). Notably, the in vivo anti-tumor efficacy achieved by the combined RIT and PTT in Group 6 appeared to be remarkably better than the predicted addictive effect (Figure 5f). Hematoxylin and eosin (H\&E) staining and terminal deoxynucleotidyl transferase-mediated dUTP nick end labeling (TUNEL) staining of tumor slices further demonstrated that the combined PTT and RIT induced by SWNT@PDA-131I-PEG (Group 6) showed the most significant damage to tumor cells and resulted in the highest level of tumor cell apoptosis (Figure 6a and 6b). This remarkable synergistic therapeutic effect by Group 6 could be attributed to the mild PTT effect, which although could not directly ablate tumors, would improve intra-tumor blood flow and relieve tumor hypoxia, so as to overcome hypoxia-associated radiation resistance for solid tumors.[36]

It is known that although raw CNT could induce 
toxic effects, $[8,37,38]$ SWNTs with short lengths and biocompatible coatings would be not obviously toxic in vitro and in vivo.[39, 40] Therefore, we examined potential toxic side effect of such combined PTT and RIT with SWNT@PDA-131I-PEG. During our in vivo experiments, no significant body weight drop was observed (Supporting Information Figure S5). In addition, histology analysis of major organs from mice at 14 days after i.v. injection of SWNT@PDA-131I-PEG and laser treatment indicated no significant organ damage (Supporting Information Figure S5). These results demonstrated that our SWNT@PDA-131I-PEG sample was no noticeably toxic to the treated mice in the experimental period.

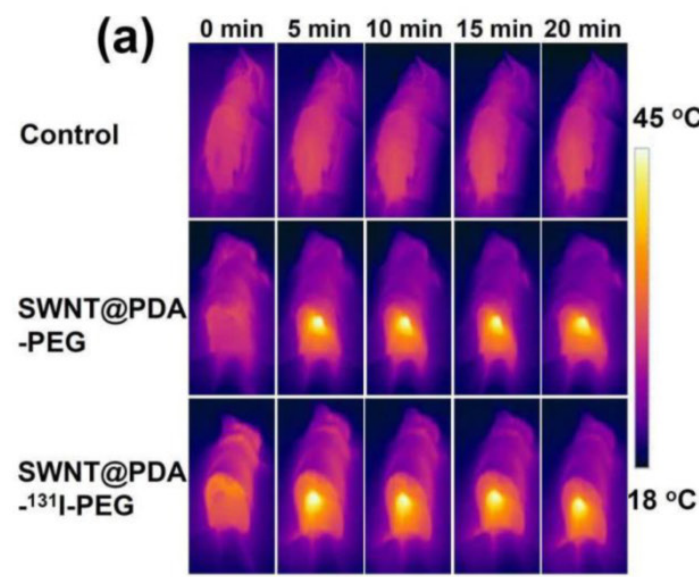

(b)
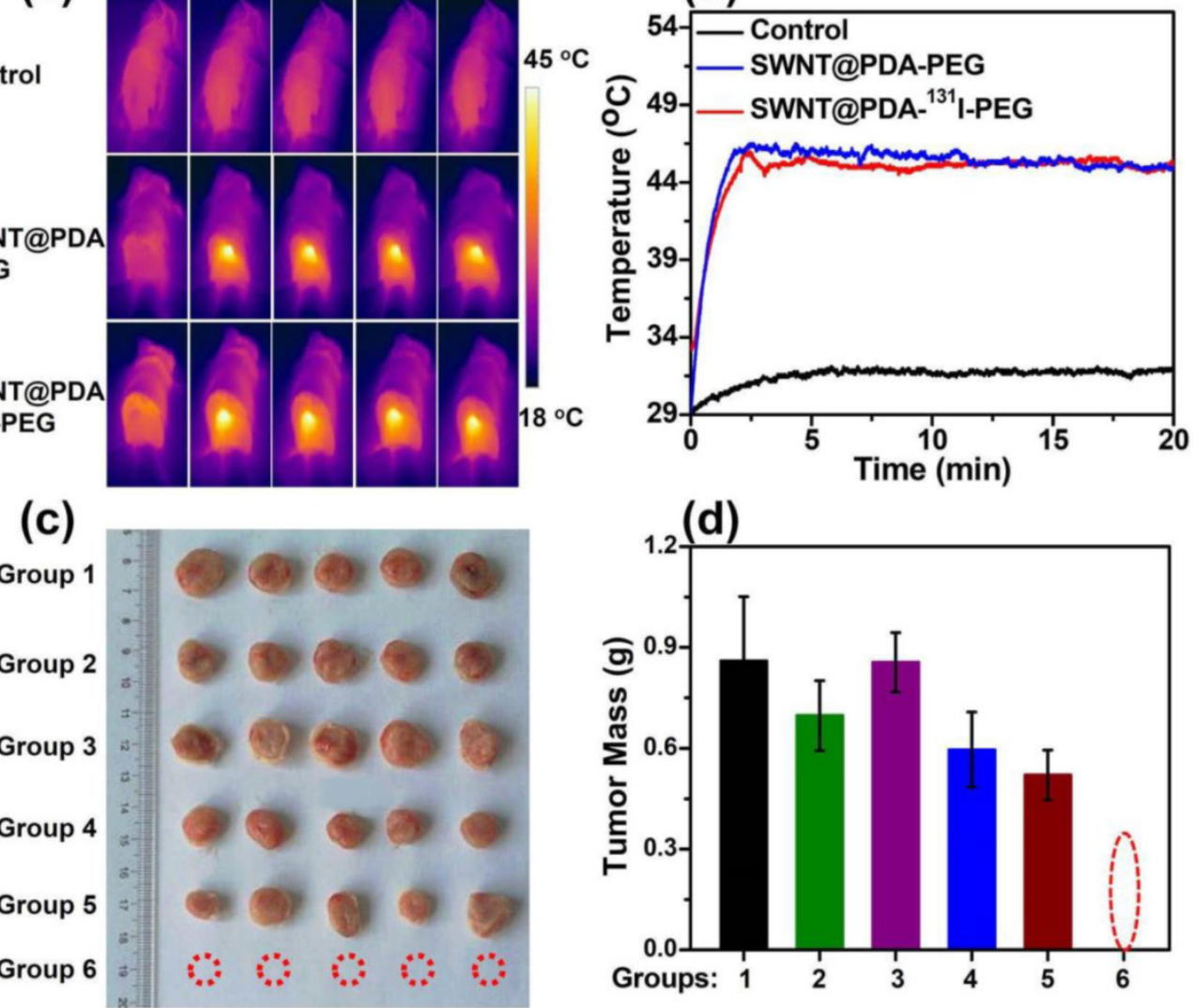

(e)


Figure 5. In vivo combined therapy with SWNT@PDA-13I|-PEG. (a) IR thermal images of 4T1 tumor-bearing mice with or without injection of SWNT@PDA-13II-PEG ([SWNT]:4.3 mg/kg, $200 \mu \mathrm{Ci}$ of ${ }^{131}$ per mouse), under the $808 \mathrm{~nm}$ laser irradiation (about $0.6 \mathrm{~W} \mathrm{~cm}^{-2}$ ). (b) Temperature change curves of tumors monitored by the IR thermal camera during laser irradiation. (c) Photos of the tumors collected from different groups of mice at day 14 after different treatments. Note that in the combined therapy group, all of the tumors were completely eliminated after treatment. (d) The tumors collected from all the groups were weighted 14 days after various treatments. (e) Tumor volume growth curves of different groups of mice after various treatments. The temperature was kept at about $46{ }^{\circ} \mathrm{C}$ for 20 minutes by NIR irradiation. (f) Relative tumor volume of different groups after various treatments. Group 1: Control; Group 2: SWNT@PDA-PEG; Group 3: Free 131; Group 4: SWNT@PDA-PEG + Laser; Group 5: SWNT@PDA-131|-PEG; Group 6: SWNT@PDA-131|-PEG + Laser. The predicted addictive effect was calculated by multiplying the relative tumor volume ratio in group 4 (PTT only) with that in group 5 (RIT only). Statistical analysis was performed using the Student's two-tailed t-test $(* * * \mathrm{p}<0.001)$. 


\section{(a)}

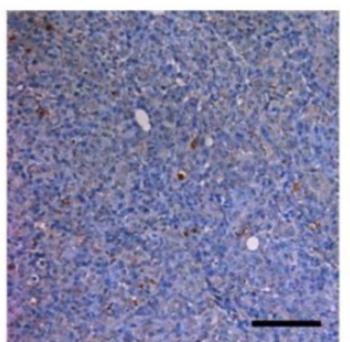

Group 1

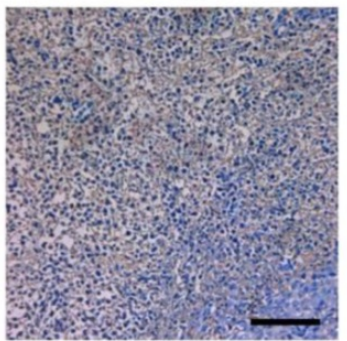

Group 4

(b)

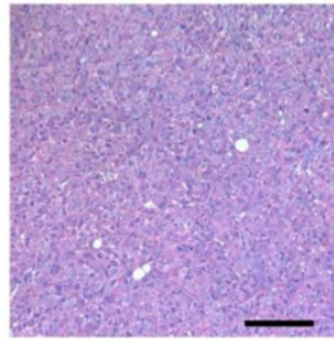

Group 1

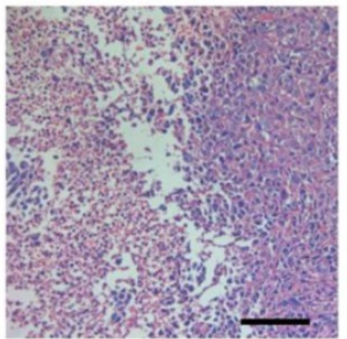

Group 4

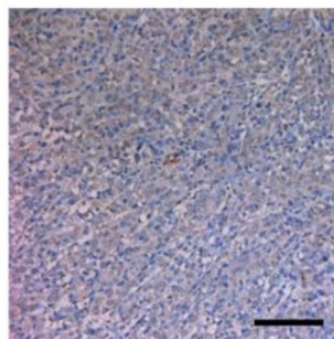

Group 2

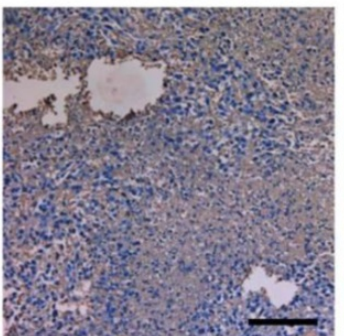

Group 5

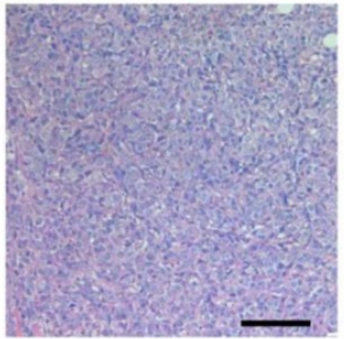

Group 2

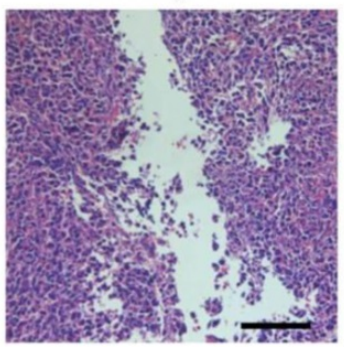

Group 5

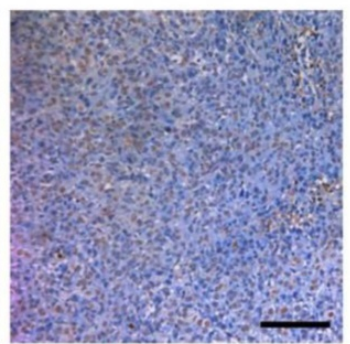

Group 3

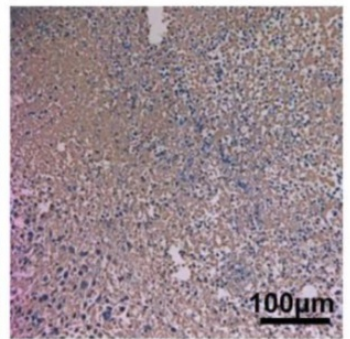

Group 6

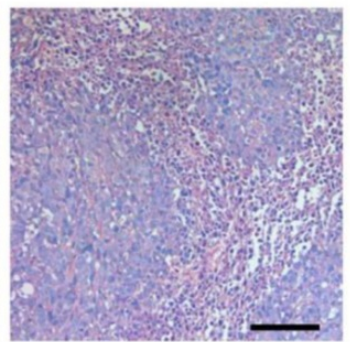

Group 3

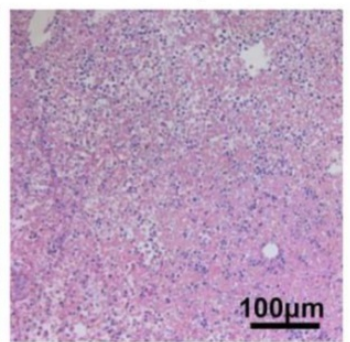

Group 6

Figure 6. H\&E staining and TUNEL staining of tumor slices. (a) TUNEL staining of the tumor slices taken at day 2 post the initiation of treatments. The combined internal RT with PTT offered remarkable synergistic therapeutic effect in inhibiting the tumor growth. (b) H\&E stained images of the tumor slices taken at day 2 post the initiation of treatments. Group 1: Control; Group 2: SWNT@PDA-PEG; Group 3: Free 131|; Group 4: SWNT@PDA-PEG + Laser; Group 5: SWNT@PDA-131|-PEG; Group 6: SWNT@PDA-131|-PEG + Laser.

\section{Conclusion}

In summary, we have developed a multifunctional nanoplatform based on PDA coated SWNTs with PEG modification. The PDA shell on SWNTs not only enables chelation with $\mathrm{Mn}^{2+}$ to enhance T1 / T2 bimodal MR imaging, but also allows labeling with ${ }^{131}$ I to deliver RIT for cancer therapy. As illustrated by T1 / T2 MR imaging and further confirmed by ex vivo biodistribution to track 131I, efficient tumor accumulation of those nanotubes is vividly observed. Taking advantages of the strong inherent NIR absorbance of SWNTs, NIR-induced PTT is then conducted in combination with ${ }^{131}$ I-based RIT using SWNT@PDA-131I-PEG, achieving a remarkable synergistic therapeutic effect compared to PTT alone or RIT alone. Therefore, an interesting theranostic nanoplatform based on SWNTs is developed in this work, useful for multimodal imaging guided combination therapy of cancer. In addition, our results also suggest the great advantages of PDA coating as a general biocompatible surface 
functionalization strategy to offer coated nanomaterials with highly integrated functionalities promising in biomedicine.

\section{Supplementary Material}

Supplementary figures.

http://www.thno.org/v06p1833s1.pdf

\section{Acknowledgements}

This work was partially supported by the National Natural Science Foundation of China (51572180, 81420108022, 81272143), the National "973" Program of China (2012CB932601), a Project Funded by the Priority Academic Program Development of Jiangsu Higher Education Institutions, and the Collaborative Innovation Center of Suzhou Nano Science and Technology (Nano-CIC), the Suzhou Municipal Science and Technology Project (SYS201565), Key Laboratory of Suzhou (SZS201307), Suzhou Clinical Medicine Center (Szzxj201505).

\section{Competing Interests}

The authors have declared that no competing interest exists.

\section{References}

1. Chen Z-Y, Wang Y-X, Lin Y, Zhang J-S, Yang F, Zhou Q-L, et al. Advance of molecular imaging technology and targeted imaging agent in imaging and therapy. BioMed research international. 2014; 2014.

2. Liu T, Shi S, Liang C, Shen S, Cheng L, Wang C, et al. Iron oxide decorated MoS2 nanosheets with double PEGylation for chelator-free radiolabeling and multimodal imaging guided photothermal therapy. ACS nano. 2015; 9: 950-60.

3. Zhong J, Wen L, Yang S, Xiang L, Chen Q, Xing D. Imaging-guided high-efficient photoacoustic tumor therapy with targeting gold nanorods. Nanomedicine: Nanotechnology, Biology and Medicine. 2015; 11: 1499-509.

4. Zhong J, Yang S, Wen L, Xing D. Imaging-guided photoacoustic drug release and synergistic chemo-photoacoustic therapy with paclitaxel-containing nanoparticles. Journal of Controlled Release. 2016; 226: 77-87.

5. Xu X, Ho W, Zhang X, Bertrand N, Farokhzad O. Cancer nanomedicine: from targeted delivery to combination therapy. Trends in molecular medicine. 2015; 21: 223-32.

6. Gong H, Cheng L, Xiang J, Xu H, Feng L, Shi X, et al. Near-Infrared Absorbing Polymeric Nanoparticles as a Versatile Drug Carrier for Cancer Combination Therapy. Advanced Functional Materials. 2013; 23: 6059-67.

7. Miao W, Shim G, Kim G, Lee S, Lee H-J, Kim YB, et al. Image-guided synergistic photothermal therapy using photoresponsive imaging agent-loaded graphene-based nanosheets. Journal of Controlled Release. 2015; 211: $28-36$.

8. Liu Z, Davis C, Cai W, He L, Chen X, Dai H. Circulation and long-term fate of functionalized, biocompatible single-walled carbon nanotubes in mice probed by Raman spectroscopy. Proceedings of the National Academy of Sciences of the United States of America. 2008; 105: 1410-5.

9. Liu J, Wang C, Wang X, Wang X, Cheng L, Li Y, et al. Mesoporous Silica Coated Single-Walled Carbon Nanotubes as a Multifunctional Light-Responsive Platform for Cancer Combination Therapy. Advanced Functional Materials. 2015; 25: 384-92.

10. Liu X, Tao H, Yang K, Zhang S, Lee ST, Liu Z. Optimization of surface chemistry on single-walled carbon nanotubes for in vivo photothermal ablation of tumors. Biomaterials. 2011; 32: 144-51.

11. Tasis D, Tagmatarchis N, Bianco A, Prato M. Chemistry of carbon nanotubes. Chemical reviews. 2006; 106: 1105-36.

12. Bianco A, Kostarelos $\mathrm{K}$, Prato M. Applications of carbon nanotubes in drug delivery. Current opinion in chemical biology. 2005; 9: 674-9.

13. Guo C, Al-Jamal WT, Toma FM, Bianco A, Prato M, Al-Jamal KT, et al. Design of Cationic Multiwalled Carbon Nanotubes as Efficient siRNA Vectors for Lung Cancer Xenograft Eradication. Bioconjugate chemistry. 2015; 26: 1370-9.

14. Kim YK, Na HK, Kim S, Jang H, Chang SJ, Min DH. One-Pot Synthesis of Multifunctional Au@ Graphene Oxide Nanocolloid Core@ Shell Nanoparticles for Raman Bioimaging, Photothermal, and Photodynamic Therapy. Small. 2015; 11 : 2527-35.
15. Choi Y, Kim S, Choi MH, Ryoo SR, Park J, Min DH, et al. Highly biocompatible carbon nanodots for simultaneous bioimaging and targeted photodynamic therapy in vitro and in vivo. Advanced Functional Materials. 2014; 24: 5781-9.

16. Miao W, Shim G, Lee S, Oh Y-K. Structure-dependent photothermal anticancer effects of carbon-based photoresponsive nanomaterials. Biomaterials. 2014; 35 : 4058-65

17. Wang X, Wang C, Cheng L, Lee ST, Liu Z. Noble metal coated single-walled carbon nanotubes for applications in surface enhanced Raman scattering imaging and photothermal therapy. J Am Chem Soc. 2012; 134: 7414-22.

18. Wang C, Ma X, Ye S, Cheng L, Yang K, Guo L, et al. Protamine Functionalized Single-Walled Carbon Nanotubes for Stem Cell Labeling and In Vivo Raman/Magnetic Resonance/Photoacoustic Triple-Modal Imaging. Advanced Functional Materials. 2012; 22: 2363-75.

19. Zhang Y, Franklin NW, Chen RJ, Dai H. Metal coating on suspended carbon nanotubes and its implication to metal-tube interaction. Chemical Physics Letters. 2000; 331: 35-41.

20. Coleman JN, Khan U, Blau WJ, Gun'ko YK. Small but strong: a review of the mechanical properties of carbon nanotube-polymer composites. Carbon. 2006; 44: 1624-52

21. Liu Y, Ai K, Liu J, Deng M, He Y, Lu L. Dopamine-Melanin Colloidal Nanospheres: An Efficient Near-Infrared Photothermal Therapeutic Agent for In Vivo Cancer Therapy. Advanced Materials. 2013; 25: 1353-9.

22. Zhong X, Yang K, Dong Z, Yi X, Wang Y, Ge C, et al. Polydopamine as a Biocompatible Multifunctional Nanocarrier for Combined Radioisotope Therapy and Chemotherapy of Cancer. Advanced Functional Materials. 2015; 25: 7327-36.

23. Liu Y, Ai K, Lu L. Polydopamine and its derivative materials: synthesis and promising applications in energy, environmental, and biomedical fields. Chemical reviews. 2014; 114: 5057-115.

24. Lin L-S, Cong Z-X, Cao J-B, Ke K-M, Peng Q-L, Gao J, et al. Multifunctional Fe3O4@ polydopamine core-shell nanocomposites for intracellular mRNA detection and imaging-guided photothermal therapy. ACS nano. 2014; 8: 3876-83

25. Chen Y, Ai K, Liu J, Ren X, Jiang C, Lu L. Polydopamine-based coordination nanocomplex for $\mathrm{T} 1 / \mathrm{T} 2$ dual mode magnetic resonance imaging-guided chemo-photothermal synergistic therapy. Biomaterials. 2016; 77: 198-206.

26. Zhang X, Liu T, Sreekumar T, Kumar S, Moore VC, Hauge RH, et al. Poly (vinyl alcohol)/SWNT composite film. Nano letters. 2003; 3: 1285-8.

27. Kam NWS, O'Connell M, Wisdom JA, Dai H. Carbon nanotubes as multifunctional biological transporters and near-infrared agents for selective cancer cell destruction. Proceedings of the National Academy of Sciences of the United States of America. 2005; 102: 11600-5.

28. Fei B, Qian B, Yang Z, Wang R, Liu WC, Mak CL, et al. Coating carbon nanotubes by spontaneous oxidative polymerization of dopamine. Carbon. 2008; 46: 1795-7.

29. Fan Q, Cheng K, Hu X, Ma X, Zhang R, Yang M, et al. Transferring biomarker into molecular probe: melanin nanoparticle as a naturally active platform for multimodality imaging. J Am Chem Soc. 2014; 136: 15185-94.

30. Song G, Liang C, Gong H, Li M, Zheng X, Cheng L, et al. Core-Shell MnSe@ Bi2Se3 Fabricated via a Cation Exchange Method as Novel Nanotheranostics for Multimodal Imaging and Synergistic Thermoradiotherapy. Advanced Materials. 2015; 27: 6110-7.

31. Yang Y, Liu J, Liang C, Feng L, Fu T, Dong Z, et al. Nanoscale Metal-Organic Particles with Rapid Clearance for MR Imaging Guided Photothermal Therapy. ACS nano. 2016.

32. Moloto N, Moloto M, Kalenga M, Govindraju S, Airo M. Synthesis and characterization of MnS and MnSe nanoparticles: Morphology, optical and magnetic properties. Optical Materials. 2013; 36: 31-5.

33. Dong Z, Gong H, Gao M, Zhu W, Sun X, Feng L, et al. Polydopamine Nanoparticles as a Versatile Molecular Loading Platform to Enable Imaging-guided Cancer Combination Therapy. Theranostics. 2016; 6: 1031-42.

34. Mi P, Kokuryo D, Cabral H, Wu H, Terada Y, Saga T, et al. A pH-activatable nanoparticle with signal-amplification capabilities for non-invasive imaging of tumour malignancy. Nature nanotechnology. 2016.

35. Lee N, Cho HR, Oh $\mathrm{MH}$, Lee $\mathrm{SH}$, Kim K, Kim BH, et al. Multifunctional $\mathrm{Fe} 3 \mathrm{O} 4 / \mathrm{TaO} \times$ Core/Shell Nanoparticles for Simultaneous Magnetic Resonance Imaging and X-ray Computed Tomography. Journal of the American Chemical Society. 2012; 134: 10309-12.

36. Song $G$, Liang $C$, Yi $X$, Zhao $Q$, Cheng $L$, Yang $K$, et al. Perfluorocarbon-Loaded Hollow Bi2Se3 Nanoparticles for Timely Supply of Oxygen under Near-Infrared Light to Enhance the Radiotherapy of Cancer. Advanced Materials. 2016.

37. Sayes CM, Liang F, Hudson JL, Mendez J, Guo W, Beach JM, et al. Functionalization density dependence of single-walled carbon nanotubes cytotoxicity in vitro. Toxicology letters. 2006; 161: 135-42.

38. Liu Z, Chen K, Davis C, Sherlock S, Cao O, Chen X, et al. Drug delivery with carbon nanotubes for in vivo cancer treatment. Cancer Res. 2008; 68: 6652-60.

39. Liu Z, Tabakman S, Welsher K, Dai H. Carbon Nanotubes in Biology and Medicine: In vitro and in vivo Detection, Imaging and Drug Delivery. Nano Res. 2009; 2: 85-120.

40. Soleyman R, Hirbod S, Adeli M. Advances in the biomedical application of polymer-functionalized carbon nanotubes. Biomaterials science. 2015; 3: 695-711. 